ARTICLE

https://doi.org/10.1038/s41467-018-07832-4

\title{
Pressure-driven phase transitions and reduction of dimensionality in 2D silicon nanosheets
}

Gil Chan Hwang (10 1, Douglas A. Blom², Thomas Vogt ${ }^{3}$, Jaejun Lee ${ }^{4}$, Heon-Jin Choi ${ }^{4}$, Sen Shao ${ }^{5}$, Yanming $\mathrm{Ma}^{5,6}$ \& Yongjae Lee (1) ${ }^{1,7}$

In-situ high-pressure synchrotron X-ray powder diffraction studies up to $21 \mathrm{GPa}$ of CVDgrown silicon 2D-nanosheets establish that the structural phase transitions depend on size and shape. For sizes between 9.3(7) $\mathrm{nm}$ and 15.2(8) $\mathrm{nm}$ we observe an irreversible phase transition sequence from I (cubic) $\rightarrow \mathrm{II}$ (tetragonal) $\rightarrow \mathrm{V}$ (hexagonal) during pressure increase and during decompression below $8 \mathrm{GPa}$ the emergence of an X-ray amorphous phase. High-angle annular dark field scanning transmission electron microscopy (HAADFSTEM) and atomic force microscopy (AFM) images of this X-ray amorphous phase reveal the formation of significant numbers of 1D nanowires with aspect ratios $>10$, which are twinned and grow along the <111> direction. We discovered a reduction of dimensionality under pressure from a $2 \mathrm{D}$ morphology to a $1 \mathrm{D}$ wire in a material with a diamond structure. MD simulations indicate the reduction of thermal conductivity in such nanowires.

\footnotetext{
${ }^{1}$ Department of Earth System Sciences, Yonsei University, Seoul 03722, Korea. ${ }^{2}$ NanoCenter \& Department of Chemical Engineering, University of South Carolina, Columbia, SC 29208, USA. ${ }^{3}$ NanoCenter \& Department of Chemistry \& Biochemistry, University of South Carolina, Columbia, SC 29208 , USA. ${ }^{4}$ Department of Materials Science and Engineering, Yonsei University, Seoul 03722, Korea. ${ }^{5}$ State Key Lab of Superhard Materials \& Innovation Center for Computational Physics Methods and softwares, College of Physics, Jilin University, 130012 Changchun, China. ${ }^{6}$ International Center of Future Science, Jilin University, 130012 Changchun, China. ${ }^{7}$ Center for High Pressure Science and Technology Advanced Research, 201203 Shanghai, China. Correspondence and requests for materials should be addressed to Y.L. (email: yongjaelee@yonsei.ac.kr)
} 
S ilicon is an abundant, low-cost, non-toxic, environmentally friendly, biocompatible, and ubiquitous chemical element used in our daily lives and industrial applications ${ }^{1}$. At ambient conditions it crystallizes in a cubic diamond structure with space group $F d \overline{3} m$, and its structural behavior with temperature $^{2-7}$, coefficients of thermal expansion ${ }^{4,8-10}$ and negative thermal expansion (NTE) at low temperatures ${ }^{11,12}$ have been well established. Changes of silicon's crystal structure at high pressures revealed metallic structures with $\mathrm{Si}$ in 6-fold, 8-fold, and 12-fold coordination ${ }^{13}$. Various allotropes including framework structures have been studied in recent years ${ }^{14}$. Amorphous, semiconducting silicon $(a-S i)$ made by chemical or physical vapor deposition is generally believed to have a four-coordinated, nonperiodic continuous random network (CRN) with 5-membered, 6-membered, and 7-membered rings of tetrahedra. Introduced by Zachariasen $^{15}, a-S i$ is metastable with respect to crystalline silicon which has only 6-membered rings. Reduced density functions of CRN models made using bond-swapping algorithms ${ }^{16,17}$ and framework relaxation ${ }^{18,19}$ match experimental high-energy X-ray, neutron ${ }^{20}$ and electron diffraction data ${ }^{21}$. However, as pointed out by Treacy and Borisenko ${ }^{22}$, reduced density functions cannot unequivocally distinguish between different CRN models. Other models for $a$-Si structures are the paracrystalline model of Hosemann and Baggchi ${ }^{23}$ and the microcrystallite model of Turnbull and Polk ${ }^{24}$ which posit that short-range ordered material at length scales of a few nanometers are subjected mainly to strain gradients in the paracrystalline model or rotational disorder in the microcrystallite model. Fluctuation electron microscopy reveals the existence of 1-2 $\mathrm{nm}$ regions of high crystallinity in different samples referred to as $a-\mathrm{Si}^{25-27}$. The short-range order at the nanometer scale found in diffraction experiments of many nanomaterials results in them being labeled "X-ray amorphous". However, electron microscopy and in particular high-angle annular dark field scanning transmission electron microscopy (HAADF-STEM) has established the presence of well-ordered structural regions with short-range translational symmetry ${ }^{28}$. This motivates HAADF-STEM investigations of highly disordered materials found after high-pressure X-ray diffraction experiments which often reveal few or no sharp X-ray and/or neutron diffraction peaks.

The structures and properties of distinct silicon nanomaterials such as zero-dimensional, one-dimensional, and two-dimensional quantum dots, nanowires and nanosheets (NS) are well established ${ }^{29,30}$. 2D NS materials have thicknesses less than $2 \mathrm{~nm}$ and reveal $\langle 110\rangle$ zone axes, often with edges of defects oriented along $<111>^{31}$. We explored the high-pressure chemistry of 2D Si NS and found that the phase transition pressures depend on the average crystallite size and shape. We observe that below $8 \mathrm{GPa}$ during decompression large amounts of $2 \mathrm{D}$-materials with crystallite sizes smaller than $15 \mathrm{~nm}$ transform into $\mathrm{x}$-ray amorphous materials which when imaged using STEM after pressure release contain large amounts of $1 \mathrm{D}$ nanowires. Si nanowires are an important class of nanomaterials used as biosensors for the detection of metal ions, nucleic acids, and viruses ${ }^{32}$. Their dimensionality strongly impacts properties such as thermal conductivity 33,34 .

Fabrication and synthesis of 1D Si nanowires is achieved using top-down approaches such as electron-beam or nanoprint lithography or bottom-up techniques based on vapor-liquid-solid (VLS) or oxide-assisted growth ${ }^{35}$. The contamination of Au used as a catalyst in VLS synthesis ${ }^{36}$ in Si nanowires, the presence of $\mathrm{SiOx}$ layers after oxide-assisted growth as well as a better control of the aspect ratio, twinning, and defects are current challenges for Si 1D-nanowire synthesis. We believe that the pressure-driven reduction in dimensionality of nanomaterials established here for silicon 2D-nanosheets made by chemical vapor deposition (CVD) is a fabrication method that should lead to more high-pressure investigations of other $2 \mathrm{D}$ nanomaterials as it opens a route to high purity materials with many potential applications in sensing, thermal electric devices and solar energy conversion ${ }^{37}$.

\section{Results and Discussion}

Ambient conditions. As synthesized silicon nanosheets were characterized by SEM, AFM, TEM, and STEM as shown in Fig. 1. The SEM and AFM images in Fig. 1a, b confirm the 2D nature of the as-grown materials. The HAADF STEM data in Fig. 1c, d show the typical dumbbell structure of the diamond cubic $\mathrm{Si}$ phase in a $<110>$ zone axis orientation. The majority of the assynthesized silicon nanosheets ( $\mathrm{Si}$ NS) were found to have a $<110>$ orientation with extended defects along $<111>$. Unit-cell parameters of the silicon nanoscale materials at ambient condition were derived using XRD data measured on samples mounted inside quartz capillaries $(\varnothing 0.5 \mathrm{~mm})$ (Supplementary Fig. 1, Supplementary Table 1). The (111), (220), (311), (400), and (331) Bragg peaks up to $48^{\circ}$ in $2 \theta\left(\mathrm{Mo}_{\mathrm{K}} \mathrm{Ka}_{1}, \lambda=0.070932(1) \mathrm{nm}\right)$ (Supplementary Fig. 1) were used to determine the unit-cell volumes to be $0.1584(1), 0.1600(1)$, and $0.1606(1) \mathrm{nm}^{3}$, for the SiNS15.2, Si-NS12.8, and Si-NS9.3 samples, respectively. Using the peak width of the (111) reflection, respective crystallite sizes were derived using the Scherrer equation ${ }^{38}$. The crystallite sizes for the Si-NS15.2, Si-NS12.8, and Si-NS9.3 samples were 15.2(4), 12.8(8), and $9.3(7) \mathrm{nm}$, respectively (Fig. 2a, Supplementary Fig. 1). It appears that there is a minimum in crystallite size and a concomitant maximum in unit cell volume when the CVD reaction time is $45 \mathrm{~min}$ (Fig. 2a, Supplementary Table 1).

Pressure-induced phase transition and recovery of X-ray amorphous silicon. In bulk silicon, the transition sequence during compression has been reported to be $\mathrm{I} \rightarrow \mathrm{II} \rightarrow \mathrm{V} \rightarrow \mathrm{VI} \rightarrow \mathrm{VII}$ up to about $50 \mathrm{GPa}^{39}$, whereas during decompression the transitions found were $\mathrm{V} \rightarrow(\mathrm{V}+\mathrm{II}) \rightarrow \mathrm{II} \rightarrow(\mathrm{II}+\mathrm{III}) \rightarrow \mathrm{III}^{40,41}$. Depending on the decompression speed the recovered phases were either phase $\mathrm{I}^{42}$, III, XI ${ }^{42}$, or an X-ray amorphous phase $\mathrm{s}^{43}$. The high-pressure VI, VII, and X phases are observed at pressures larger than $34 \mathrm{GPa}^{44}$. In particular, phase $\mathrm{X}(f c c)$ is only stable between 78.3 and $230 \mathrm{GPa}^{45,46}$

We performed high-pressure diffractions experiments up to 20.5(1), 21.0(1), and 20.5(1) GPa for the Si-NS9.3, Si-NS12.8, and Si-NS15.2 samples, respectively. The high-pressure silicon phases have previously been established as $\mathrm{Si}-\mathrm{I}(\mathrm{Fd} \overline{3} \mathrm{~m}$, cubic, $Z=8)$, SiII ( $I 4_{1} / a m d$, tetragonal, $\left.Z=4\right)$, Si-V $(P 6 / \mathrm{mmm}$, hexagonal, $Z=$ $1)$, and X-ray amorphous silicon $(a-\mathrm{Si})$. The observed phase transition sequence of the $2 \mathrm{D}$ silicon nanosheet samples is $\mathrm{I} \rightarrow$ $\mathrm{II} \rightarrow \mathrm{V}$ under compression and $\mathrm{V} \rightarrow \mathrm{II} \rightarrow a$-Si during decompression for Si-NS9.3, Si-NS12.8, and Si-NS15.2. In contrast to bulk silicon samples, we did not detect any evidence for the presence of the Kasper phase (Si-III) and lonsdaleite (Si-IV) at pressures up to $9 \mathrm{GPa}^{47-49}$. The well-established (111) twinning in silicon has an $\mathrm{ABAB}$ stacking sequence and can be understood as a fragment of lonsdaleite (Si-IV). The orientation relationships $(111)_{\text {Si-I }} \|$ $(001)_{\text {Si-IV }} \|(100)_{\text {Si-III }}$ were established in electron microscopy studies ${ }^{49}$. However, larger domain sizes which would also diffract in an X-ray powder diffraction experiment require the transfer of the hexagonal motif from a template such as a hexagonal GaP nanowire upon which one can then epitaxially grow an extended Si shell ${ }^{50}$.

The high-pressure phase transitions for the Si-NS9.3, SiNS12.8, and Si-NS15.2 samples are irreversible and in all cases Xray amorphous $a$-Si is found below $8 \mathrm{GPa}$ during decompression. The onset pressure of individual phase transitions, the region where two phases coexist, and the hysteresis upon decompression 

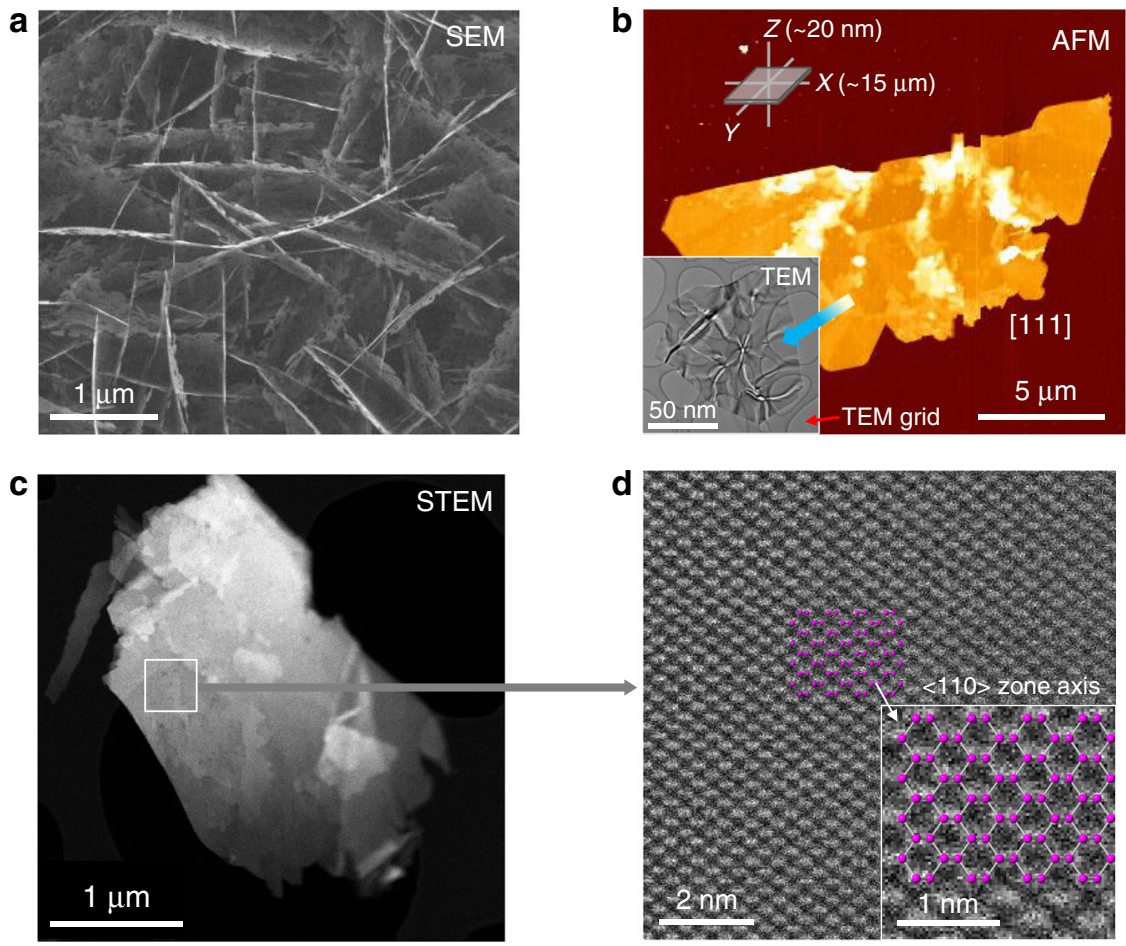

Fig. 1 Images of silicon nanosheets. a SEM image of a silicon nanosheet (Si-NS). b AFM, and TEM images of the silicon particle of [111] direction, about 20 $\mathrm{nm}$ thick. c, d STEM images of Si-NSs showing surfaces with silicon dumbbells along the $\langle 110\rangle$ zone axis

differ slightly but not systematically and are summarized in Fig. 3 (Supplementary Table 2 and Supplementary Figs. 2-4).

The Si-Si distance and hence the unit cell volume of Si NS vary in response to pressure ${ }^{51-53}$. The detailed pressure-dependent changes of the normalized unit cell volumes for the Si-NS samples given in Supplementary Figs. $2 \mathrm{~b}-4 \mathrm{~b}$ and the bulk moduli of various phases point to the rich high-pressure chemistry and its dependence on crystallite size and shape. During decompression from 19.0(1) GPa, the formation of $a$-Si using porous silicon was first reported by Deb et al. ${ }^{54}$ and pressure-induced amorphization of Si following a compression/decompression cycle was subsequently investigated ${ }^{55,56}$. Using wafer indentation experiments, Gogotsi et al. studied the phase transitions of silicon as a function of decompression speed and observed that silicon transformed to an amorphous phase near $3 \mathrm{GPa}$, below which it reverted to Si-I or Si-III ${ }^{57,58}$. Recent theoretical work predicted that metastable silicon following decompression would transform to Si-III ${ }^{59}$. We have confirmed by STEM imaging after 12 months that these nanowires are stable after pressure release and no reversible phase transition back to $2 \mathrm{D}$-nanosheets occurs. The recovered X-ray amorphous silicon phases after compressing and decompressing silicon $2 \mathrm{D}$-NS are therefore inconsistent with results on bulk $\mathrm{Si}$ and point to the important role of dimensionality and size of 2DSi under pressure.

HAADF STEM images from the X-ray amorphous materials in Fig. 4 all reveal significant amounts of well-ordered 1D nanowires (see also rotational STEM images in Fig. 5). HAADF STEM images of the starting materials are shown in the left most column of Fig. 4 for reference. After being subjected to high pressure and decompression below $8 \mathrm{GPa}$, the low magnification HAADF STEM images in the second column indicate the amount of 1-D nanowires formed. The long axis of the nanowires is along $<111>$ and the nanowires are typically in a $<110>$ zone axis orientation relative to the electron beam. Significant twinning of the nanowires along $<111>$ can be seen in the far right column of Fig. 4 for all the Si NS samples. It appears that under decompression the defects of the 2D-Si nanosheets which predominantly were located on edges pointing in the $<111>$ direction grew to become the long axis of the $1 \mathrm{D}$ nanowires. We found aspect ratios above 50 with observed widths of about $15 \mathrm{~nm}$ and lengths near one micron. This is in the size range of what can be done using top-down wafer-scale patterning 60 . Our samples obtained from the gaskets after high-pressure X-ray experiments (Fig. 4) were not sonicated nor were any other attempts made to further isolate and separate the Si nanowires. The only important step was to dissolve the silicon oil used as a pressure-transmitting medium as it degrades in the electron beam. We observed regions on our microscopy grid where $1 \mathrm{D}$-Si nanowires are well separated from the bulk of the material, which will become advantageous when using micromanipulators. Important future work would be to attempt to replicate our work done in a diamond anvil cell using nano-indentation.

It has been shown that nano-indentation can transform crystalline $\mathrm{Si}$ in wafers into various high-pressure polymorphs and, after decompression, into an amorphous $\mathrm{Si}$ phase ${ }^{61}$. Aberration-corrected STEM offers unique opportunities to characterize and distinguish "X-ray amorphous" phases as it does not rely on the presence of materials with extended translation symmetry. Our work points to a route for top-down pressure-driven nanowire synthesis using 2D-Si NS which might be applicable to other nanomaterials. The pressure-driven formation of the isolated Si nanowires has also been confirmed by AFM imaging (Fig. 6). Our unequivocal detection of highdensity 1D Si nanowires in these X-ray amorphous phases made by compressing $2 \mathrm{D}$ nanosheets establishes a synthesis process for materials with applications in solid-state electronics and photovoltaics technologies.

Molecular dynamics calculations of thermal conductivity. One physical consequence of reducing the dimensionality to $1 \mathrm{D}-\mathrm{Si}$ nanowires is a reduction of the thermal conductivity along the 

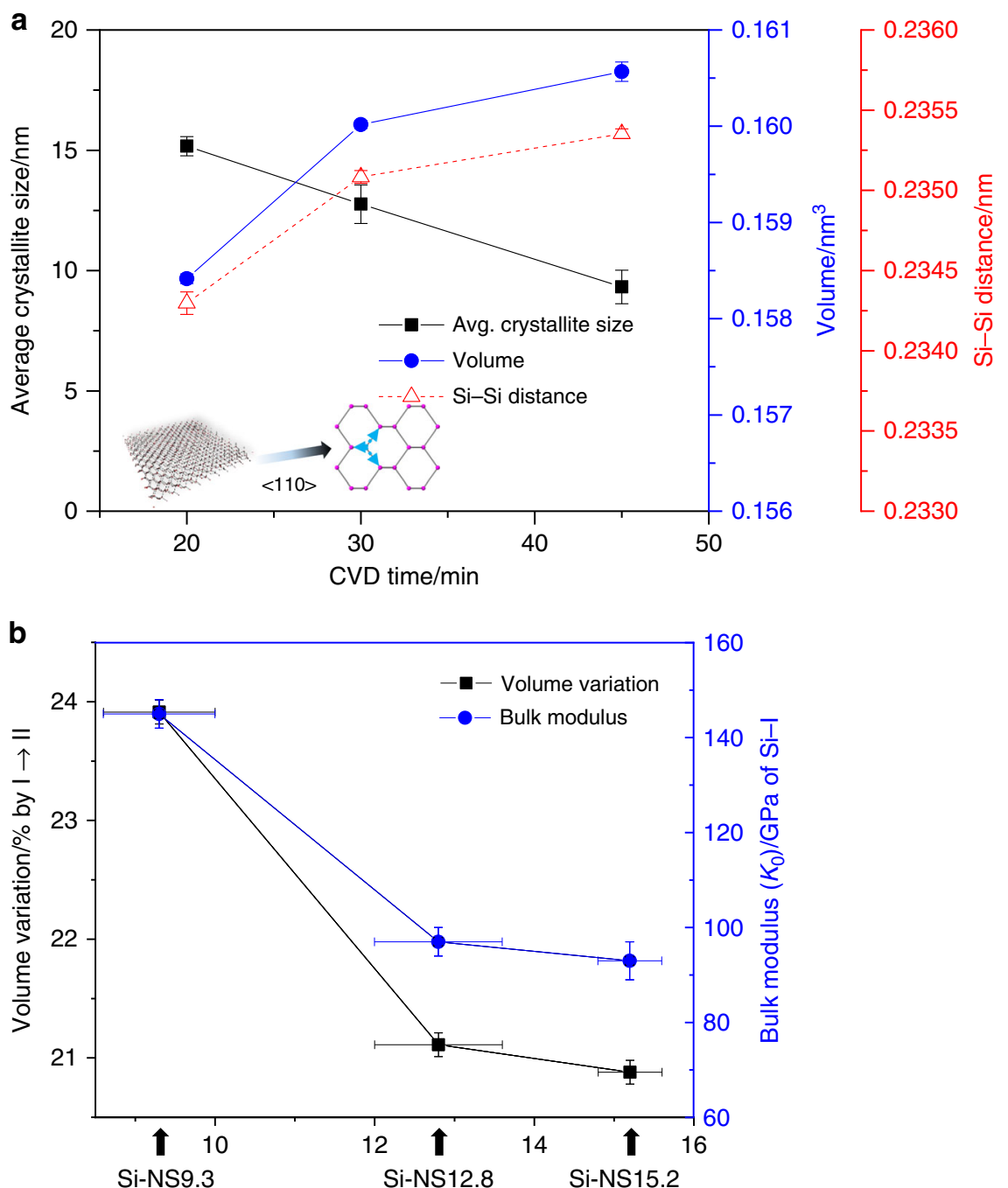

Average crystallite size/nm

Fig. 2 Physical parameters and pressure response of Si-nanosheets. a Crystallite size and unit cell volume as a function of CVD reaction time. Using the peak width of the (111) reflection, crystallite sizes were derived using the Scherrer equation. $\mathbf{b}$ Bulk moduli of nanoscale Si-I phase and the degree of volume contraction upon Si-II transition as a function of crystallite size (arrows)

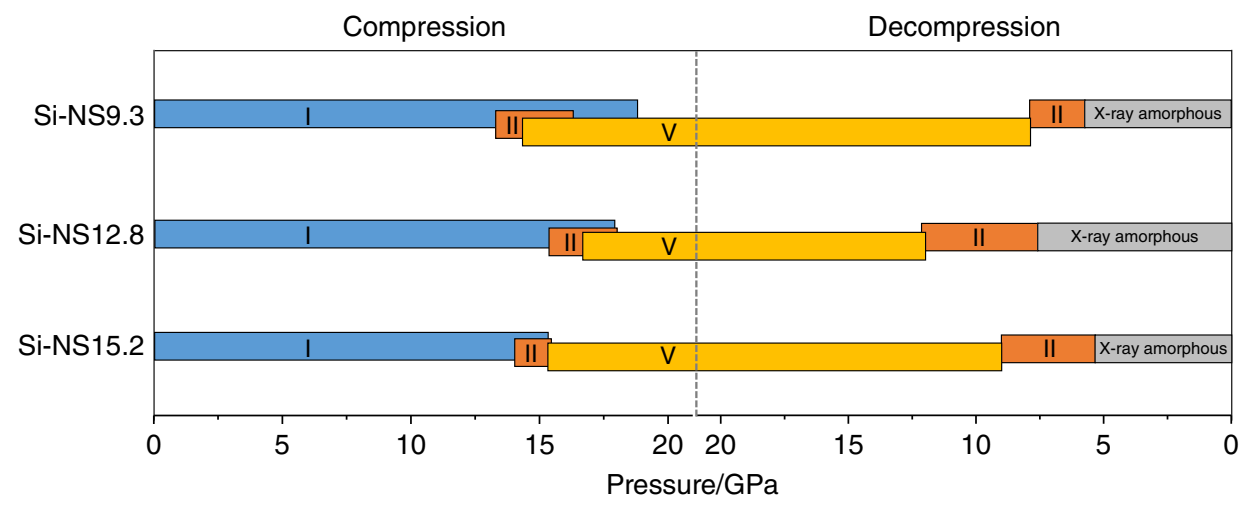

Fig. 3 Transition sequence of silicon nanosheets. Phase transitions for Si-NS9.3, Si-NS12.8, and Si-NS15.2 during compression up to 20.5(1), 21.0(1), and 20.5(1) GPa, respectively, and then decompression

direction of the wire. The calculated thermal conductivity of bulk silicon is $226.54 \pm 18.17 \mathrm{~W} / \mathrm{mK}$ and in good agreement with the value of $226.07 \pm 2.86 \mathrm{~W} / \mathrm{mK}$ given by Zhou ${ }^{62}$ using the same potential and method. As shown in Fig. 7, the thickness of the 2D silicon nanosheets has a minute effect on the thermal conductivity resulting in an average value of $106 \mathrm{~W} / \mathrm{mK}$, while the thermal conductivity along the $<100>$ direction for a Si-NW was found to be $39.62 \pm 4.51 \mathrm{~W} / \mathrm{mK}$. Previous theoretical work 
2D nanosheet
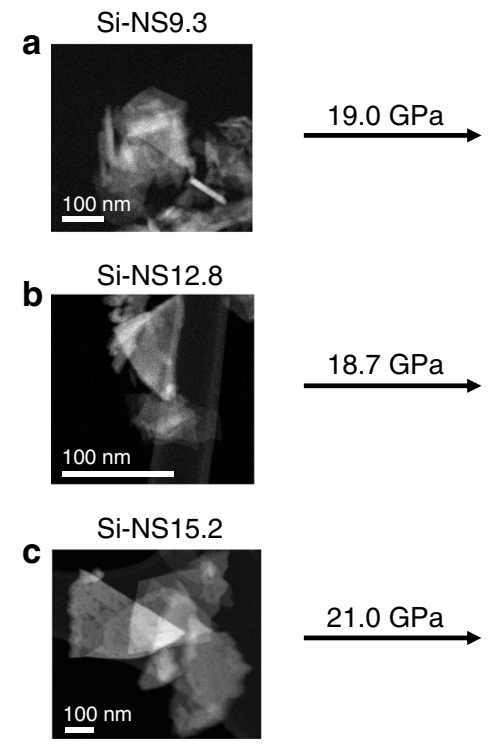

Dimensional reduction
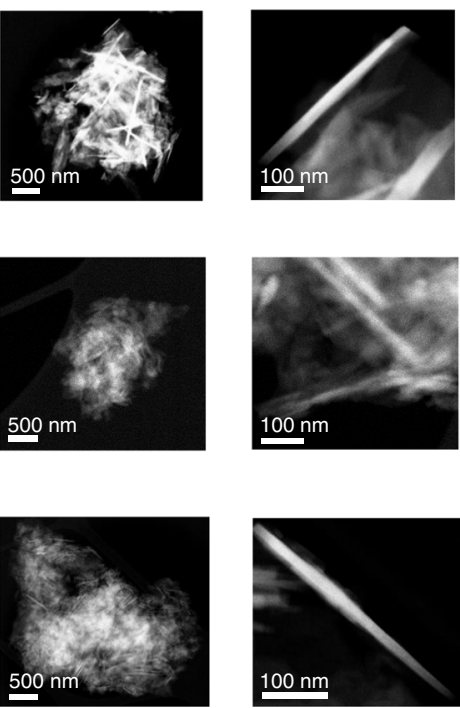

1D nanowire
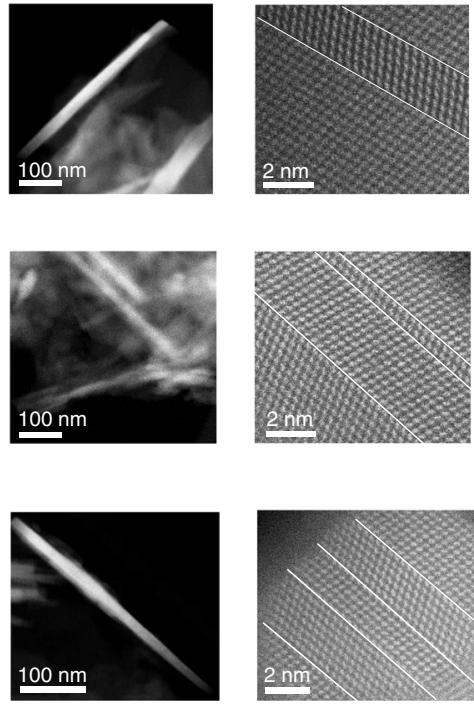

Fig. 4 HAADF-STEM images of Si-nanowires formed from Si-nanosheets. 2D-Si-nanosheets of different crystallite sizes are seen on the left. a Si-NS9.3. b Si-NS12.8. c Si-NS15.2 Increasing magnification HAADF-STEM images of nanowires found in X-ray amorphous materials subsequent to high-pressure and complete decompression. White lines are the twinning planes $\{111\}$ for Si. The 2D Si-nanosheets transformed to 1D Si-nanowires during decompression at room temperature

a

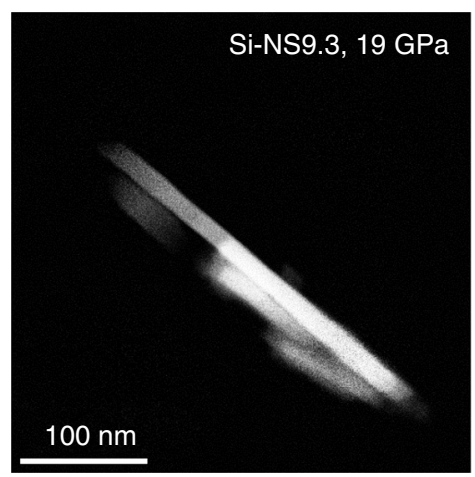

b
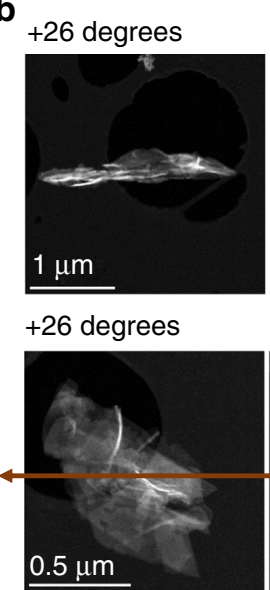

0 degrees

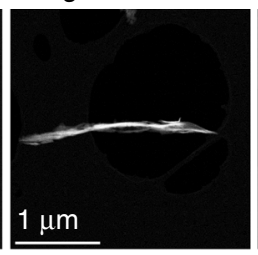

0 degrees

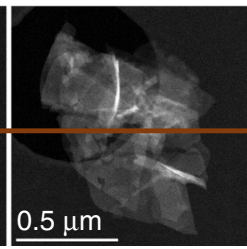

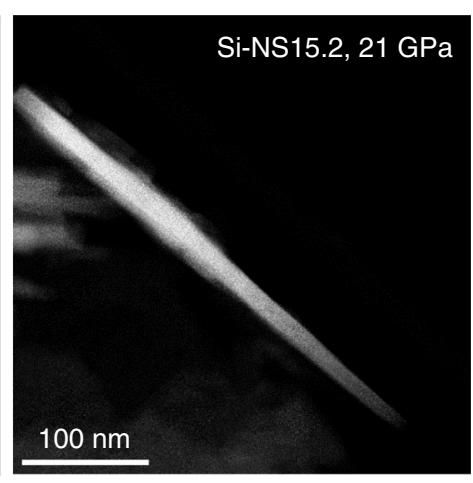

-26 degrees

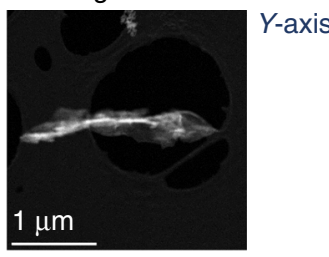

-26 degrees

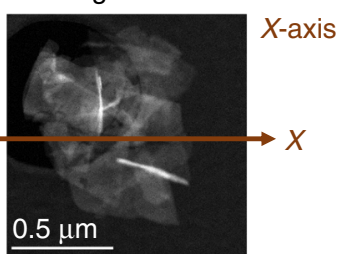

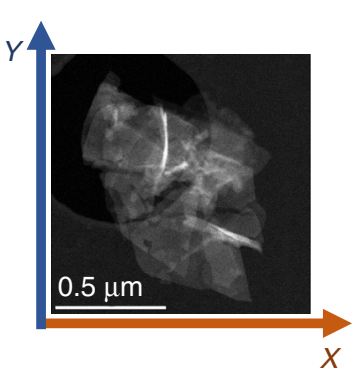

Fig. 5 HAADF-STEM images of isolated 1D Si-nanowires. a Isolated Si-NS9.3_19 GPa and Si-NS15.2_21 GPa. b The rotational HAADF-STEM images of the 1D Si-nanowires 

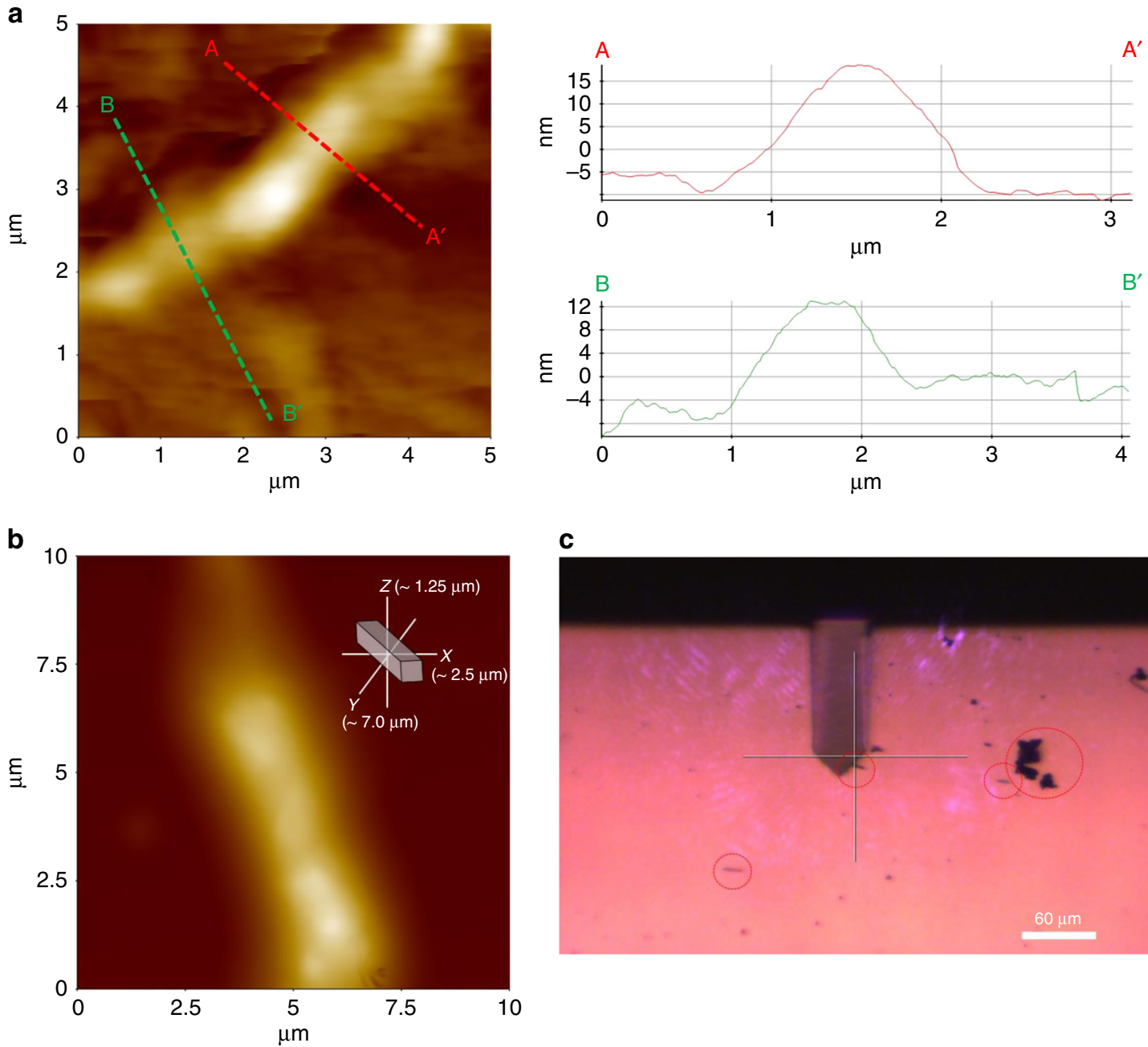

C

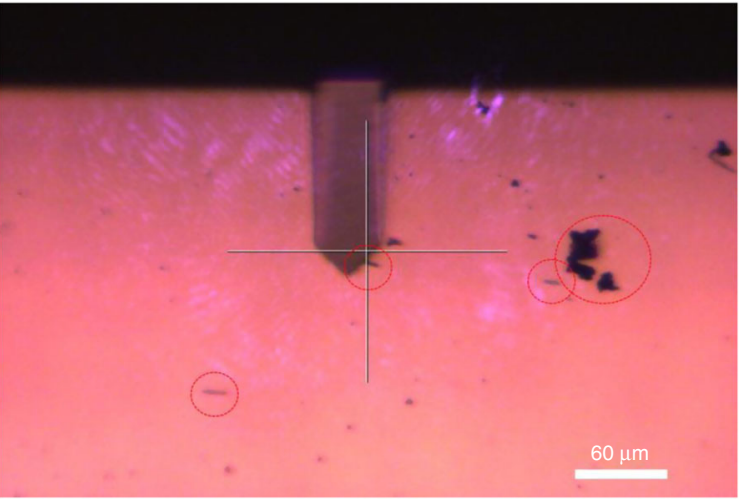

Fig. 6 AFM images of isolated 1D Si-nanowires. a Si-NS9.3_21 GPa and its 1-dimensional height profile sections. b Another AFM image of Si-NS9.3_21 GPa with its dimension. c Optical image of nanowires (circled) dispersed on a glass substrate (Park Systems, XE-100)

revealed that the thermal conductivities along the $<110\rangle^{62}$ and $<100>$ direction $^{63}$ for $\mathrm{Si}-\mathrm{NW}$ are $64.5 \pm 4.5$ and $43 \pm 10 \mathrm{~W} / \mathrm{mK}$, respectively. The thermal conductivities of $\mathrm{Si}-\mathrm{NWs}$ are significantly below that of Si-NS and bulk materials, indicating that the thermal conductivity reduces significantly with the reduction of dimensionality. This reduction of dimensionality is a promising way to increase the ZT coefficient which is a dimensionless figure of merit defined as $\mathrm{ZT}=S^{2} T \sigma / \kappa$, with $S$ being the Seebeck coefficient, $\kappa$ the thermal conductivity, and $\sigma$ the electrical conductivity at temperature $T$. Therefore, silicon nanomaterials and other nanowires with a diamond structure made by using pressure have the potential to be applied to improved thermoelectrics.

\section{Methods}

Sample synthesis. The synthetic silicon nanosheet samples used in this study were prepared by Kim et al. ${ }^{31} \mathrm{~A} c$-plane sapphire substrate was used in CVD to produce Si-NS12.8 and Si-NS15.2 nanosheets while a quartz substrate was used for Si-

NS9.3. In this paper the sample name is given by NS (nanosheet) followed by the average crystallite in nanometer. The sapphire substrate was first subjected to a wet cleaning process and then placed in the center of a furnace through which Ar and $\mathrm{H}_{2}$ flowed at $300 \mathrm{sccm}$ (standard cubic centimeters per minute) as the temperature was increased to $1273 \mathrm{~K}$ over a period of $25 \mathrm{~min}$. Once the temperature reached $1273 \mathrm{~K}$, the flow rate was increased to $3000 \mathrm{sccm}$. Pure samples of Si-NS15.2, SiNS12.8, Si-NS9.3 were obtained by bubbling $\mathrm{H}_{2}$ at $20 \mathrm{sccm}$ in liquefied $\mathrm{SiCl}_{4}$ for 20 $\min , 30 \mathrm{~min}, 45 \mathrm{~min}$, respectively ${ }^{31}$.

Electron imaging. We identified the crystallite types of $15 \mu \mathrm{m}$ long and $20 \mathrm{~nm}$ thick samples using field emission scanning electron microscopy (FE-SEM), atomic force microscope (AFM), and transmission electron microscopy (TEM). The sheet size varied depending on the $\mathrm{H}_{2}$ bubbling time and/or amount. The surface was either the [111] or [110] plane depending on the synthesis conditions (Fig. 1) ${ }^{31}$. An AFM image using a Nanowizard I (JPK instruments) with tapping mode was obtained. Abberation-corrected STEM (JEOL JEM-2100F) was performed at 200 $\mathrm{kV}$ in the high angle annular dark field mode. Imaging with an SEM (JEOL JSM$7100 \mathrm{~F})$ was done at $15 \mathrm{kV}(\times 10,000)$ without coating.

Phase identification and crystallite size. X-ray powder diffraction was performed in transmission method to qualitatively analyze each 2D-Si-NS sample using a MicroMax-007HF (Rigaku Corp.) equipped with a rotating anode Mo-Ka $(\lambda=0.070932(1) \mathrm{nm})$ with multilayer optics (VariMax-Mo, Rigaku) and an imaging plate (IP) detector $\left(R\right.$-axis $\mathrm{IV}^{++}$, Rigaku). The X-ray generator was operated at $1.2 \mathrm{~kW}(50 \mathrm{kV}, 24 \mathrm{~mA})$, and the $\mathrm{X}$-ray beam size was reduced to $100 \mu \mathrm{m}$ by using a pin-hole collimator. The sample-to-detector distance was $120 \mathrm{~mm}$ and the typical exposure time was $10 \mathrm{~min}$. Samples were rotated between 0 and $180^{\circ}$ using a quartz capillary with a diameter of $0.5 \mathrm{~mm}$. IPanalyzer v3.551 and Crystalclear v2.0 programs were used to calibrate and process the $2 \mathrm{D}$ diffraction images ${ }^{64,65}$. $\mathrm{LaB}_{6}$ (NIST SRM 660c, $a=0.4157044(8) \mathrm{nm}$ ) standard powder was used for the instrument calibration and initial condition.

The crystallite size $(D)$ was calculated using the Scherrer equation, $D_{(111)}(\mathrm{nm})=$ $0.94 \lambda / \beta \cos \theta^{38}$, where $\lambda$ is X-ray wavelength, $\theta$ (radian) is diffraction angle, and $\beta$ (radian) is $\beta_{\text {sample broadening }} \beta_{\text {instrumental broadening (SRM LaB6 } 660 \mathrm{c}, \mathrm{FWHM}=0.2042 \mathrm{~nm} \text { ) at }}$ the full width at half maximum (FWHM) which was calculated using profile-fitting method with a pseudo-Voigt function in the CMPR software ${ }^{66}$. The Scherrer constant used was 0.94 for cubic symmetry. The peak broadening was measured using a Si standard and the same instrumental conditions. We would like to point out that the crystallite sizes obtained from diffraction measurements and those observed in STEM images are based on completely different physical mechanisms ${ }^{67}$ and in most cases will not agree. However, in-situ crystallite 


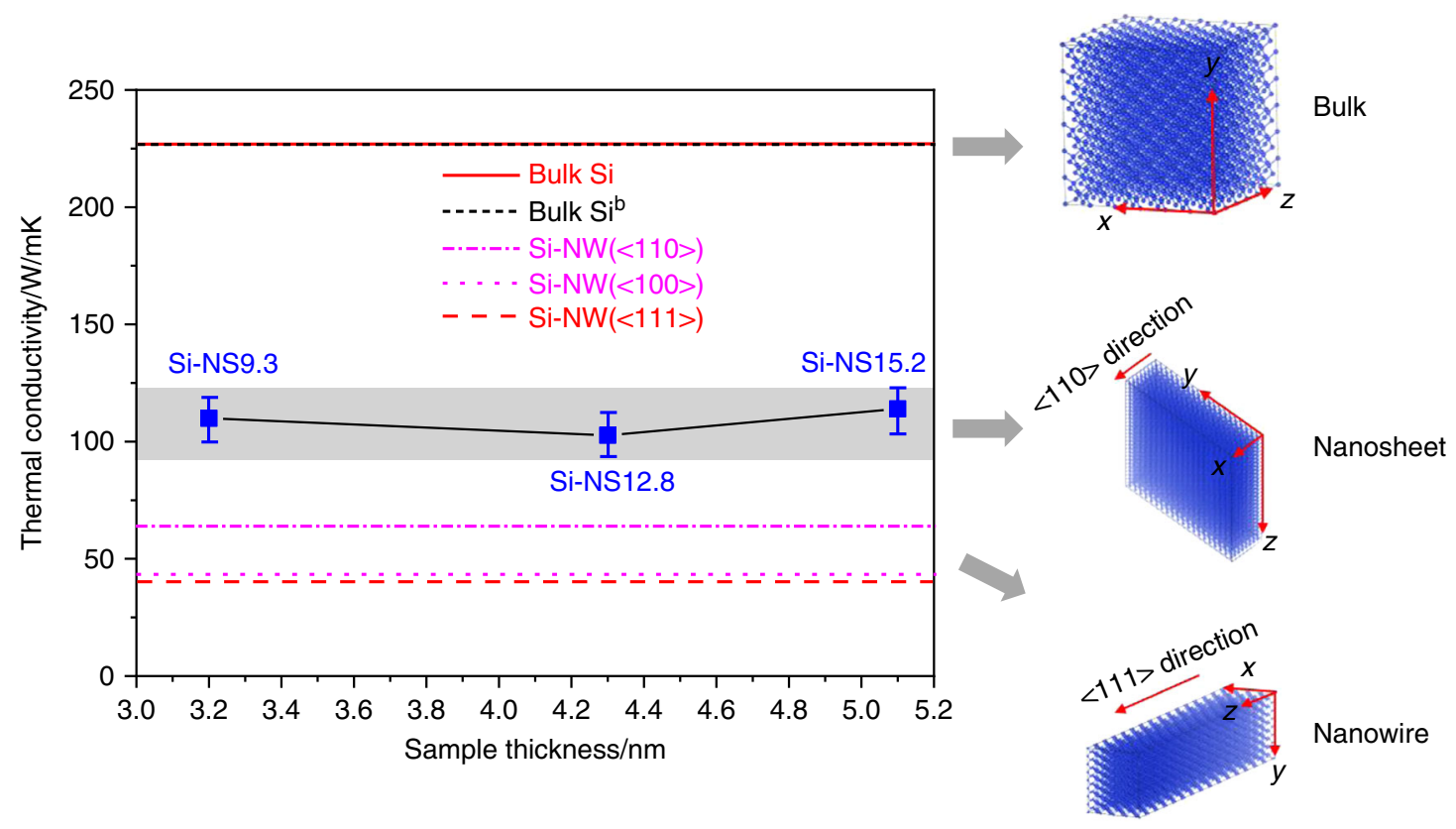

Fig. 7 Calculated thermal conductivity of bulk silicon and Si-nanostructures. The thermal conductivity of bulk Si, bulk Sib (predicted by Zhou62), <111> direction Si-NW, $<110>$ surface Si-NW (predicted by Zhou ${ }^{62}$ ), $<100>$ direction Si-NW (predicted by Donadio ${ }^{63}$ ), Si-NS9.3, Si-NS12.8, and Si-NS15.2. Gray area is the fluctuant range of the thermal conductivity for nanosheets. The model systems studied in this work for bulk silicon, silicon nanosheet with $<110>$ zone axis orientation, and silicon nanowire of which the large dimension is along <111> direction, are shown as insets

determinations in a diamond anvil cell provide us with a qualitative guidance of the crystallite size which we cannot be obtained using imaging methods.

High-pressure experiments. High-pressure X-ray diffraction experiments were performed at the Pohang Accelerator Laboratory (PAL). The Si-NS12.8 and SiNS15.2 samples were measured at the PLS-II 9 A beamline at PAL. Passing a double crystal monochromator equipped with bent $\mathrm{Si}$ (111) and $\mathrm{Si}(311)$ crystals and a pinhole, monochromatic X-rays with a wavelength of $0.0622(1) \mathrm{nm}$ were used. The angle-dispersive X-ray diffraction data were measured using a CCD detector (Rayonix SX165, $2048 \times 2048$ pixels, exposure time of 150 s). The SiNS9.3 sample was measured at the PLS-II 5 A beamline at PAL. Monochromatic Xray with a wavelength of $0.0692(1) \mathrm{nm}$ was used in combination with an image plate detector (marXperts mar345, $3000 \times 3000$ pixels, exposure time $\sim 150 \mathrm{~s}$ ). $\mathrm{A} \mathrm{LaB}_{6}$ (NIST SRM 660c, $a=0.4157(1) \mathrm{nm}$ ) standard powder was used for the instrument calibration.

As a high-pressure device we used a symmetric-type diamond-anvil cell (SDAC). The culet diameter of the diamond anvil (type I) was approximately $400 \mu \mathrm{m}$. Stainless steel T301 (thickness $0.25 \mathrm{~mm}$ ) was used as a gasket material after indentation to about $80 \mu \mathrm{m}$. The sample chamber in the gasket was formed using an electric discharge machine (EDM; Holozoic products) and was about $150 \mu \mathrm{m}$ in diameter. The pressure-transmitting medium used to generate hydrostatic conditions on the sample was a methanol: ethanol: water mixture (16:3:1 by volume) in the case of Si-NS12.8 and Si-NS15.2, and silicone oil (10 cSt) in the case of Si-NS9.368,69. The pressure was increased from ambient to ca. $8.0 \mathrm{GPa}$ in ca. $0.5 \mathrm{GPa}$ increments at room temperature. The sample was equilibrated at each pressure for about $15 \mathrm{~min}$ before the XRD measurement. The pressure was calculated from the shift of the ruby $(>40 \mu \mathrm{m}$ in size $) \mathrm{R} 1$ emission line and the established formulae of $P( \pm 0.05 \mathrm{GPa})=$ $1904 / 7.665\left[\left(1+\left(\Delta \lambda / \lambda_{0}\right)\right)^{7.665}-1\right]^{70}$. The lattice parameters were refined by applying the whole-pattern fitting LeBail method using the GSAS suite of programs ${ }^{71,72}$. Bulk moduli from normalized volume $\left(V / V_{0}\right)$ were calculated using the Murnaghan equation of state $\left.\left(V / V_{0}=\left[1+K^{\prime} P / K_{0}\right]^{-1 / K^{\prime}}\right), K^{\prime}=(\Delta K / \Delta P)_{P=0}=4\right)$ or $P=3 /$ $2 K_{0}\left[\left(V / V_{0}\right)^{-7 / 3}-\left(V / V_{0}\right)^{-5 / 3}\right]\left(1+3 / 4\left(K_{0}^{\prime}-4\right)\left[\left(V / V_{0}\right)^{-2 / 3}-1\right]\right), K_{0}=-V(\Delta P / \Delta V)_{P=0}$, $K_{0}{ }^{\prime}=(\Delta K / \Delta P)_{P=0}{ }^{73,74}$, which was integrated within the EOSFIT and the OriginLab programs ${ }^{75,76}$.

Scanning transmission electron microscopy. The HAADF-STEM images of materials before after compression were taken using a JEOL JEM $2100 \mathrm{~F}$ equipped with a CEOS-corrector to minimize spherical aberration of the electron probe. The powder samples were meticulously and painstakingly cleaned with hexane to remove the silicone oil used as a pressure-transmission fluid in the X-ray experiments before Z-contrast imaging. Failure to do so will lead to rapid decomposition of the silicone oil and subsequently the samples will degrade under the $200 \mathrm{kV}$ electron beam. Cleaned and dried powders were loaded onto holey carbon-coated copper grids. The electron probe used at $200 \mathrm{kV}$ to image the sample had a 24 mrad convergence angle. The images were recorded with a Fischione Model
3000 detector and a camera length such that the detector spanned between 75 and $178 \mathrm{mrad}$. The scanning acquisition was synchronized to the $60 \mathrm{~Hz}$ AC electrical power to minimize $60 \mathrm{~Hz}$ noise in the images. A pixel dwell time of $15.8 \mu \mathrm{s}$ was used.

Molecular dynamics simulation. All theoretical MD simulations were performed using the Tersoff potential ${ }^{77}$ for interatomic interactions in an equilibrium molecular dynamics (EMD) calculation based on the Green-Kubo formula ${ }^{78}$ in a large-scale atomic/molecular massively parallel simulator environment

(LAMMPS) $^{79}$. The model system we studied in this work is shown in Fig. 7. We chose a simulation cell of $\mathrm{Lx} \times \mathrm{Ly} \times \mathrm{Lz}=3.265 \times 3.265 \times 3.265 \mathrm{~nm}^{3}$ for bulk silicon. In the nanosheet simulations, the thicknesses along the $x$ direction were set to 3.2 $\mathrm{nm}, 4.3 \mathrm{~nm}$, and $5.1 \mathrm{~nm}$, for the samples NS9.3, NS12.8, and NS15.2,

respectively, and the areas perpendicular to the $x$ direction are used for the same size of $\mathrm{Ly} \times \mathrm{Lz}=11.54 \times 11.42 \mathrm{~nm}^{2}$. We employ free boundary condition in the $x$ direction and periodic boundary conditions in the $\mathrm{y}$ and $\mathrm{z}$ directions. For the $\mathrm{Si}$ nanowire (Si-NW), the cross-section ( $x y$ plane) is chosen as $\mathrm{Lx} \times \mathrm{Ly}=5.395 \times$ $5.535 \mathrm{~nm}^{2}$ and free boundary condition is used along the $x$ and $y$ directions. Furthermore, periodic boundary conditions are employed along the $z$ direction and the length of $\mathrm{Lz}$ was chosen to be $23.56 \mathrm{~nm}$. A time step of $1 \mathrm{fs}$ was employed in our simulations. The bulk and 2D-nanosheets are relaxed in the NVT ensemble at 300 $\mathrm{K}$ for 500 ps to obtain the optimized structures, and then the heat current is calculated in the NVE ensemble. A longer $7 \mathrm{~ns}$ run is needed for Si-NW in the NVT ensemble to reach the equilibrium structure. NVT (constant crystallite, constant volume, and constant temperature) is a canonical ensemble. NVE (constant crystallites, constant volume, and constant total energy) is a micro-canonical ensemble.

\section{Data availability}

The data that support the findings of this study are available within the article and its Supplementary Information file or available from the corresponding author upon reasonable request.

Received: 14 June 2018 Accepted: 27 November 2018

Published online: 21 December 2018

\section{References}

1. Jaroniec, M. Silicon beyond the valley. Nat. Chem. 1, 166 (2009).

2. Debye, P. \& Scherrer, P. Interferenzen an regellos orientierten Teilchen im Röntgenlicht. I. Phys. Z 17, 277-283 (1916). 
3. Jette, E. R. \& Foote, F. Precision determination of lattice constants. J. Chem. Phys. 3, 605-616 (1935).

4. Straumanis, M. E. \& Aka, E. Z. Lattice parameters, coefficients of thermal expansion, and atomic weights of purest silicon and germanium. J. Appl. Phys. 23, 330-334 (1952).

5. Parrish, W. Results of the IUCr precision lattice-parameter project. Acta Crystallogr. 13, 838-850 (1960).

6. Straumanis, M. E., Borgeaud, P. \& James, W. J. Perfection of the lattice of dislocation-free silicon, studied by the lattice-constant and density method. $J$. Appl. Phys. 32, 1382-1384 (1961).

7. Patterson, A. L. The scherrer formula for $\mathrm{x}$-ray particle size determination. Phys. Rev. 56, 978-982 (1939).

8. Dutta, B. N. Lattice constants and thermal expansion of silicon up to $900{ }^{\circ} \mathrm{C}$ by X-ray method. Phys. Status Solidi B 2, 984-987 (1962).

9. Batchelder, D. N. \& Simmons, R. O. Lattice constants and thermal expansivities of silicon and of calcium fluoride between $6^{\circ}$ and $322^{\circ} \mathrm{K}$. J. Chem. Phys. 41, 2324-2329 (1964).

10. Xing, X., Chen, J., Deng, J. \& Liu, G. Lattice constants and thermal expansion of silicon up to 900 C. Rare Met. 23, 364 (2004).

11. Shah, J. S. \& Straumanis, M. E. Thermal expansion behavior of silicon at low temperatures. Solid State Commun. 10, 159-162 (1972).

12. Biernacki, S. \& Scheffler, M. Negative thermal expansion of diamond and zinc-blende semiconductors. Phys. Rev. Lett. 63, 290-293 (1989).

13. Liu, L.-g. \& Bassett, W. A. Elements, Oxides, and Silicates: High-Pressure Phases with Implications for the Earth's Interior (Oxford Monographs on Geology and Geophysics) 1-260 (Oxford University Press, Oxford, 1986).

14. Kim, D. Y., Stefanoski, S., Kurakevych, O. O. \& Strobel, T. A. Synthesis of an open-framework allotrope of silicon. Nat. Mater. 14, 169-173 (2015).

15. Zachariasen, W. H. The atomic arrangement in glass. J. Am. Chem. Soc. 54, 3841-3851 (1932).

16. Wooten, F., Winer, K. \& Weaire, D. Computer generation of structural models of amorphous Si and Ge. Phys. Rev. Lett. 54, 1392-1395 (1985).

17. Barkema, G. T. \& Mousseau, N. High-quality continuous random networks. Phys. Rev. B 62, 4985-4990 (2000).

18. Vink, R. L. C., Barkema, G. T., van der Weg, W. F. \& Mousseau, N. Fitting the Stillinger-Weber potential to amorphous silicon. J. Non-Cryst. Solids 282, 248-255 (2001).

19. Vink, R. L. C., Barkema, G. T., Stijnman, M. A. \& Bisseling, R. H. Device-size atomistic models of amorphous silicon. Phys. Rev. B 64, 245214 (2001).

20. Laaziri, K. et al. High-energy $\mathrm{x}$-ray diffraction study of pure amorphous silicon. Phys. Rev. B 60, 13520-13533 (1999).

21. Cockayne, D. J. H. The study of nanovolumes of amorphous materials using electron scattering. Ann. Rev. Mater. Res 37, 159-187 (2007).

22. Treacy, M. M. J. \& Borisenko, K. B. The local structure of amorphous silicon. Science 335, 950 (2012).

23. Hosemann, R. \& Bagchi, S. N. Direct Analysis of Diffraction by Matter 734 (North-Holland, Amsterdam, 1962).

24. Turnbull, D. \& Polk, D. E. Structure of amorphous semiconductors. J. NonCryst. Solids 8-10, 19-35 (1972).

25. Treacy, M. M. J., Gibson, J. M. \& Keblinski, P. J. Paracrystallites found in evaporated amorphous tetrahedral semiconductors. J. Non-Cryst. Solids 231, 99-110 (1998).

26. Voyles, P. M., Gerbi, J. E., Treacy, M. M. J., Gibson, J. M. \& Abelson, J. R Absence of an abrupt phase change from polycrystalline to amorphous in silicon with deposition temperature. Phys. Rev. Lett. 86, 5514-5517 (2001).

27. Gibson, J. M., Treacy, M. M. J., Sun, T. \& Zaluzec, N. J. Substantial crystalline topology in amorphous silicon. Phys. Rev. Lett. 105, 125504 (2010).

28. Vogt, T., Blom, D. A., Jones, L. \& Buttrey, D. J. ADF-STEM imaging of nascent phases and extended disorder within the Mo-V-Nb-Te-O catalyst system. Top. Catal. 59, 1489-1495 (2016).

29. Grazianetti, C., Cinquanta, E. \& Molle, A. Two-dimensional silicon: the advent of silicene. 2D Mater. 3, 012001 (2016).

30. Okamoto, H., Sugiyama, Y. \& Nakano, H. Synthesis and modification of silicon nanosheets and other silicon nanomaterials. Chem. Eur. J. 17, 9864-9887 (2011).

31. Kim, U. et al. Synthesis of Si nanosheets by a chemical vapor deposition process and their blue emissions. ACS Nano 5, 2176-2181 (2011).

32. Namdari, P., Daraee, H. \& Eatemadi, A. Recent advances in silicon nanowire biosensors: synthesis methods, properties, and applications. Nanoscale Res. Lett. 11, 406 (2016).

33. Zhang, G. \& Zhang, Y.-W. Thermal conductivity of silicon nanowires: from fundamentals to phononic engineering. Phys. Status Solidi RRL 7, 754-766 (2013).

34. Li, D. et al. Thermal conductivity of individual silicon nanowires. Appl. Phys. Lett. 83, 2934-2936 (2003).
35. Schmidt, V., Wittemann, J. V., Senz, S. \& Gösele, U. Silicon nanowires: a review on aspects of their growth and their electrical properties. Adv. Mater. 21, 2681-2702 (2009)

36. Oh, S. H. et al. Point defect configurations of supersaturated $\mathrm{Au}$ atoms inside Si nanowires. Nano Lett. 8, 1016-1019 (2008).

37. Wippermann, S. et al. High-pressure core structures of Si nanoparticles for solar energy conversion. Phys. Rev. Lett. 110, 046804 (2013).

38. Scherrer, P. Bestimmung der grosse und der inneren struktur von kolloidteilchen mittels Rontgenstrahlen. Göttinger Nachr. Gesell 2, 98 (1918)

39. Olijnyk, H., Sikka, S. K. \& Holzapfel, W. B. Structural phase transitions in Si and Ge under pressures up to $50 \mathrm{GPa}$. Phys. Lett. A 103, 137-140 (1984).

40. George, A. High pressure phases of c-Si. Prop. Cryst. Silicon 20, 104-107 (1997).

41. Hu, J. Z. \& Spain, I. L. Phases of silicon at high pressure. Solid State Commun 51, 263-266 (1984).

42. Zhao, Y.-X., Buehler, F., Sites, J. R. \& Spain, I. L. New metastable phases of silicon. Solid State Commun. 59, 679-682 (1986).

43. Gogotsi, Y., Zhou, G. H., Ku, S. S. \& Cetinkunt, S. Raman microspectroscopy analysis of pressure-induced metallization in scratching of silicon. Semicond. Sci. Technol. 16, 345-352 (2001).

44. Hull, R. Crystalline silicon. Inspec Publ. 20, 1-1016 (1999).

45. Duclos, S. J., Vohra, Y. K. \& Ruoff, A. L. Experimental study of the crystal stability and equation of state of Si to 248 GPa. Phys. Rev. B 41, 12021-12028 (1990).

46. Duclos, S. J., Vohra, Y. K. \& Ruoff, A. L. hcp to fcc transition in silicon at 78 GPa and studies to 100 GPa. Phys. Rev. Lett. 58, 775-777 (1987).

47. Wentorf, R. H. \& Kasper, J. S. Two new forms of silicon. Science 139, 338-339 (1963).

48. Kasper, J. S. \& Richards, S. M. The crystal structures of new forms of silicon and germanium. Acta Crystallogr. 17, 752-755 (1964).

49. Blank, V. D. \& Kulnitskiy, B. A. Crystallogeometry of polymorphic transitions in silicon under pressure. High Press. Res 15, 31-42 (1996).

50. Hauge, H. I. T. et al. Hexagonal silicon realized. Nano Lett. 15, 5855-5860 (2015).

51. Magomedov, M. N. Surface energy and pressure of diamond and silicon nanocrystals. J. Surf. Investig. 5, 705-711 (2011).

52. Jaccodine, R. J. Surface energy of germanium and silicon. J. Electrochem. Soc. 110, 524-527 (1963).

53. Neergaard Waltenburg, H. \& Yates, J. T. Surface chemistry of silicon. Chem. Rev. 95, 1589-1673 (1995).

54. Deb, S. K., Wilding, M., Somayazulu, M. \& McMillan, P. F. Pressure-induced amorphization and an amorphous-amorphous transition in densified porous silicon. Nature 414, 528-530 (2001).

55. Daisenberger, D. et al. Polyamorphic amorphous silicon at high pressure: Raman and spatially resolved X-ray scattering and molecular dynamics studies. J. Phy. Chem. B 115, 14246-14255 (2011).

56. McMillan, P. F. Amorphous materials: relaxing times for silicon. Nat. Mater. 3, 755-756 (2004)

57. Domnich, V. \& Gogotsi, Y. Phase transformations in silicon under contact loading. Rev. Adv. Mater. Sci. 3, 1-36 (2002).

58. Gogotsi, Y., Rosenberg, M. S., Kailer, A. \& Nickel, K. G. in Tribology Issues and Opportunities in MEMS(ed. Bhushan, B.) 431-442 (Springer, Dordrecht, 1998).

59. Zhu, Q., Oganov, A. R., Lyakhov, A. O. \& Yu, X. Generalized evolutionary metadynamics for sampling the energy landscapes and its applications. Phys. Rev. B 92, 024106 (2015).

60. Keith, J. M., Gregory, N., Shufeng, B. \& Stephen, Y. C. Wafer-scale patterning of sub-40 nm diameter and high aspect ratio ( $>50: 1)$ silicon pillar arrays by nanoimprint and etching. Nanotechnology 19, 345301 (2008).

61. Allen, D. et al. Observation of nano-indent induced strain fields and dislocation generation in silicon wafers using micro-Raman spectroscopy and white beam X-ray topography. Nucl. Instr. Methods Sect. B 268, 383-387 (2010).

62. Zhou, Y. \& Hu, M. Record low thermal conductivity of polycrystalline Si nanowire: breaking the casimir limit by severe suppression of propagons. Nano Lett. 16, 6178-6187 (2016).

63. Donadio, D. \& Galli, G. Atomistic simulations of heat transport in silicon nanowires. Phys. Rev. Lett. 102, 195901 (2009).

64. Seto, Y., Nishio-Hamane, D., Nagai, T. \& Sata, N. Development of a software suite on X-ray diffraction experiments. Rev. High Press. Sci. Technol. 20, 269-276 (2010)

65. Rigaku. CrystalClear 2.0 User Manual. 1-299 (Rigaku Co., 2009).

66. Toby, B. CMPR-a powder diffraction toolkit. J. Appl. Crystallogr. 38, 1040-1041 (2005).

67. Pyrz, W. D. \& Buttrey, D. J. Particle size determination using TEM: a discussion of image acquisition and analysis for the novice microscopist. Langmuir 24, 11350-11360 (2008). 
68. Hazen, R. M. \& Finger, L. W. Comparative Crystal Chemistry: Temperature, Pressure, Composition and the Variation of Crystal Structure. 1-248 (J. Wiley \& Sons, London, 1982).

69. Shen, Y., Kumar, R. S., Pravica, M. \& Nicol, M. F. Characteristics of silicone fluid as a pressure transmitting medium in diamond anvil cells. Rev. Sci. Instrum. 75, 4450-4454 (2004)

70. Mao, H. K., Xu, J. \& Bell, P. M. Calibration of the ruby pressure gauge to 800 kbar under quasi-hydrostatic conditions. J. Geophys. Res. 91, 4673-4676 (1986).

71. Le Bail, A., Duroy, H. \& Fourquet, J. L. Ab-initio structure determination of LiSbWO6 by X-ray powder diffraction. Mater. Res. Bull. 23, 447-452 (1988).

72. Toby, B. EXPGUI, a graphical user interface for GSAS. J. Appl. Crystallogr. 34, 210-213 (2001).

73. Murnaghan, F. D. The compressibility of media under extreme pressures. Proc Natl Acad. Sci. USA 30, 244-247 (1944).

74. Birch, F. Finite elastic strain of cubic crystals. Phys. Rev. 71, 809-824 (1947).

75. Angel, R. J. High-pressure structural phase transitions. Rev. Mineral. Geochem. 39, 85-104 (2000).

76. Angel Ross, J., Alvaro, M. \& Gonzalez-Platas, J. EosFit7c and a Fortran module (library) for equation of state calculations. Zkri 229, 405 (2014).

77. Tersoff, J. Modeling solid-state chemistry: interatomic potentials for multicomponent systems. Phys. Rev. B 39, 5566-5568 (1989).

78. Kubo, R. Statistical-mechanical theory of irreversible processes. I. General theory and simple applications to magnetic and conduction problems. J. Phys. Soc. Jpn 12, 570-586 (1957).

79. Plimpton, S. Fast parallel algorithms for short-range molecular dynamics. J. Comput. Phys. 117, 1-19 (1995).

\section{Acknowledgements}

This work was supported by the Leader Researcher program (NRF-2018R1A3B1052042) of the Korean Ministry of Science, ICT and Planning (MSIP). D.B and T.V. thank the VPR at the University of South Carolina for support operating the JEOL-2100F STEM. We also thank the support of the Korean NRF grants 2016K1A4A3914691 and 2016K1A3A7A09005244. S.S. and Y.M. are supported by the National Natural Science Foundationof China (under Grants No.11534003), Program for JLU Science andTechnology Innovative Research Team and Science Challenge Project(No.TZ2016001).

\section{Author contributions}

G.C.H. contributed to the high-pressure experiments and data analysis. J.L. performed the synthesis and initial characterization of the samples, D.A.B. measured STEM images from the as synthesized and pressure-treated samples, and S.S. performed computational calculations. Y.L. designed the research, discussed the results with T.V., H.-J.C., and Y.M. and worked on the manuscript with all authors.

\section{Additional information}

Supplementary Information accompanies this paper at https://doi.org/10.1038/s41467 018-07832-4.

Competing interests: The authors declare no competing interests

Reprints and permission information is available online at http://npg.nature.com/ reprintsandpermissions/

Journal peer review information: Nature Communications thanks the anonymous reviewers for their contributions to the peer review of this work.

Publisher's note: Springer Nature remains neutral with regard to jurisdictional claims in published maps and institutional affiliations.

(c) (i) Open Access This article is licensed under a Creative Commons Attribution 4.0 International License, which permits use, sharing, adaptation, distribution and reproduction in any medium or format, as long as you give appropriate credit to the original author(s) and the source, provide a link to the Creative Commons license, and indicate if changes were made. The images or other third party material in this article are included in the article's Creative Commons license, unless indicated otherwise in a credit line to the material. If material is not included in the article's Creative Commons license and your intended use is not permitted by statutory regulation or exceeds the permitted use, you will need to obtain permission directly from the copyright holder. To view a copy of this license, visit http://creativecommons.org/ licenses/by/4.0/.

(C) The Author(s) 2018 